the dystonia became generalized. Grimacing, dysarthria, tongue protrusion, and dysphagia with pseudobulbar palsy occurred by age 14 .

Macroscopically, the brains were unremarkable. Microscopic examination showed diffuse glial fibrillary acidic protein-immunoreactive astrocytes, and iron accumulation in neurons of the globus pallidus and substantia nigra. Additional degenerative findings included; 1) eosinophilic, rod-like cytoplasmic inclusions found in neocortical and thalamic neurons that were actin depolymerizing factor/cofilin-immunoreactive and rarely actin positive; and 2) eosinophilic spherical structures in the striatum that were actin- and actin depolymerizing factor/cofilin-positive. Aggregation of actin is a novel finding in neurodegenerative disease associated with dopa-unresponsive dystonia. (Gearing M, Juncos JL, Procaccio V et al. Aggregation of actin and cofilin in identical twins with juvenile-onset dystonia. Ann Neurol October 2002;52:465-476). (Respond: Dr Marla Gearing, Center for Neurodegenerative Disease, Whitehead Research Building, 615 Michael Street, 5th Floor, Atlanta, GA 30322).

COMMENT. An extensive aggregation of actin and actin regulatory proteins $\mathrm{ADF} /$ cofilin in twins with progressive and fatal dystonia is indicative of a dysfunction of regulatory turnover of active filaments in the cytoskeletal system. This is a novel neuropathological finding in a neurodegenerative disease causing dystonia. Other well known examples of a secondary dystonia include Wilson's disease, Hallervorden-Spatz disease, and Huntington's disease. Secondary dystonias may be complicated by parkinsonism, myoclonus, and tremor, and some are without defined pathology, as in drug-induced and occupational, and with known pathology, such as traumatic, metabolic, and vascular. Another example of secondary dystonia is the myoclonus-dystonia syndrome, with onset in childhood.

\title{
MYOCLONUS-DYSTONIA SYNDROME AND E-SARCOGLYCAN DEFICIENCY
}

Clinical and genetic findings in 9 European families with myoclonusdystonia syndrome (MDS) are reported from Ludwig-Maximilians-Universitat, Munich, Germany. In 24 affected and genetically proven patients, the clinical presentation was homogeneous, with "lightening-like" myoclonus of the neck, trunk, and upper limbs in 23 and cervical dystonia and/or writer's cramp in 13 (54\%) cases. The mean age at onset of myoclonus was 5.4 years (range $0.5-20$ years), and of dystonia 8.8 years (range 1-38 years). Myoclonus was improved by alcohol ingestion in 21 , some having severe or periodic heavy drinking. None showed progression of symptoms after age 20. Five patients had a history of panic attacks, depression, and agoraphobia. Pedigree analyses identified 8 maternal transmissions of SGCE mutations in 5 families, 7 of the mutation carriers being asymptomatic. Six novel and one known heterogeneous mutations in the gene for E-sarcoglycan (SGCE) were identified. The data confirm the role of SGCE mutations and deficiency in the pathogenesis of MDS. (Asmus F, Zimprich A, Tezenas du Montcel S et al. Myoclonus-dystonia syndrome: E-sarcoglycan mutations and phenotype. Ann Neurol October 2002;52:489-492). (Respond: Dr Gasser, Neurologische Klinik Grosshadern, Ludwig-Maximilians-Universitat, Munchen, Marchioninistrasse 15, D81337 Munchen, Germany).

COMMENT. Myoclonus-dystonia syndrome (MDS) is an autosomal dominant disorder with brief, "lightening" myoclonic jerks and cervical or brachial dystonia, with onset in childhood or early adolescence. The myoclonus is alcohol sensitive, and many develop an alcohol dependence as well as panic attacks and obsessive-compulsive disorder. In contrast to primary generalized dystonias, the 
lower limbs are rarely affected at onset in MDS, and the dystonia affects the neck muscles and hands causing neck torsion and writer's cramp. The locus for MDS is mapped to 7 q21 region, and 7 different heterogeneous mutations in the gene for E-sarcoglycan (SGCE) have now been identified. An SGCE deficiency is involved in the mechanism of MDS.

\section{NEUROCUTANEOUS SYNDROMES}

\section{LATE-ONSET GLIOMA WITH NEUROFIBROMATOSIS TYPE 1}

The frequency of symptomatic nonoptic pathway brain tumors in adolescents and adults known to have neurofibromatosis type 1 (NF1) was determined from the National Neurofibromatosis Foundation International Database (NNFFID) in a study at Washington University School of Medicine, St Louis, MO. These tumors were reported in $17(0.8 \%)$ of 2108 patients with NF1 over 10 years of age. The prevalence of brain tumors in patients with NF1 up to 50 years of age is more than 100 times greater than expected, and is significantly elevated at all ages. Of 17 patients with NF1 with adequate clinical information (10 from the NNFFID study and 7 from a National Cancer Institute (NCI) newly diagnosed adult glioma study), brain tumors were diagnosed in 4 cases between 10 and 20 years of age, 7 between 21 and 40 years of age, and in 6 at 41 years or older. Tumors were located in brainstem, cerebellum, cortical and subcortical regions. Three were pilocytic astrocytomas, 6 were grade II, 4 were grade III, and 2 were grade IV (glioblastoma multiforme) neoplasms (grades III and IV in patients older than 20). None had received chemotherapy or radiation previously. Headache was the most frequent presenting symptom (in 15), and paralysis or seizures in 3 patients each. (Gutmann DH, Rasmussen SA, Wolkenstein P et al. Gliomas presenting after age 10 in individuals with neurofibromatosis type 1 (NF1). Neurology September (1 of 2) 2002;59:759-761). (Reprints: Dr David H Gutmann, Department of Neurology, Washington University School of Medicine, Box 8111, 660 S Euclid Ave, St Louis, MO 63110).

COMMENT. Patients with NF1 are at increased risk for the development of astrocytomas beyond 10 years of age and in adult life. In older patients these tumors may be malignant grades III and IV gliomas, not always the more characteristic pilocytic astrocytoma. Population-based epidemiologic studies are recommended, but brain imaging and careful clinical follow-up should be performed in patients with NF1 of any age who present with persistent headache or neurologic abnormalities.

\section{NEUROFIBROMATOSIS TYPE 1 AND SPORADIC OPTIC GLIOMAS}

The natural history of sporadic optic gliomas was compared with that of optic gliomas associated with neurofibromatosis type 1 (NF1) in a study using a Children's Tumor Registry (CTR) and an NF1 Database (NF1DB) at St Mary's Hospital, Manchester, UK. A total of 52 cases were identified over a period of 41 years. Of 34 whose natural history was available, 31 were symptomatic. Mean ages of presentation were 4.5 and 5.1 years for NF1 and sporadic cases, respectively. Visual impairment was the presenting complaint in 22, 7 being blind in at least one eye. Recurrence occurred in 12 . Overall mortality (47\% among NF1 patients and $44 \%$ in sporadic cases) and 5 and 10 year survival rates ( $77 \%$ and $67 \%$ respectively) were similar in the two groups, but fewer NF1 patients died as a direct result of the optic glioma. Only NF1 optic glioma cases were at risk of developing a second CNS tumor. Five children (29\%) with NF1 developed second primary intracranial tumors between 7 and 32 years after initial treatment. Two 